\title{
Sound velocities in solid hydrogen under pressure
}

\author{
Yu.A. Freiman, Alexei Grechnev, and S.M. Tretyak \\ B. Verkin Institute for Low Temperature Physics and Engineering of the National Academy of Sciences of Ukraine \\ 47 Lenin Ave., Kharkov 61103, Ukraine \\ E-mail: freiman@ilt.kharkov.ua
}

Alexander F. Goncharov and Russell J. Hemley

Geophysical Laboratory, Carnegie Institution of Washington

5251 Broad Branch Road NW, Washington DC 20015, USA

Received January 18, 2013

\begin{abstract}
We present results of semi-empirical lattice dynamics calculations of the sound velocities in solid hydrogen under pressure based on the many-body intermolecular potential and first-principle density-functional theory (DFT). Both the sound velocities and elastic moduli are in excellent agreement with data from Brillouin scattering measurements while Silvera-Goldman and Hemley-Silvera-Goldman potentials tend to overestimate the sound velocity. It is shown that the stiffer is the potential the greater is overestimated the sound velocity. As was the case for equation of state and Raman-active lattice phonon calculations, the employed many-body potential works well for phases I and II (up to $140 \mathrm{GPa}$ while for higher pressures the use of the DFT is preferable.

PACS: $64.30 . J \mathrm{k}$ Equations of state of nonmetals;

67.80.F- Solids of hydrogen and isotopes;

78.30.Am Elemental semiconductors and insulators.
\end{abstract}

Keywords: solid hydrogen, sound velocity, density-functional theory.

The behavior of solid and liquid hydrogen at high pressure is one of the most fundamental problems of condense matter physics and planetary science. The central characteristics of every substance is equation of state (EOS) which is a basis for studying all physical properties of the substance, in particular for developing and examining model intermolecular potentials and testing ab initio theories. Systematic high-pressure studies of the EOS of solid hydrogens were started in the 1970s [1-6], and at present they reach the pressures up to $\sim 2$ Mbar [7-13]. The highest volume compression reached in the EOS experiments is 10.4 for solid hydrogen [13] (7.6 for solid $\mathrm{D}_{2}$ [12]), higher than for solid helium (8.4) [14]. This is an enormous change of molecular volume. For comparison, the relative change of the molar volume of every substance with temperature in the temperature range from $0 \mathrm{~K}$ to the melting point is $\sim 10 \%$, two orders of magnitude smaller.

There have been many attempts to propose the pair potential describing high-pressure properties of solid hydrogens [10,15-17]. At small pressures the Silvera-Goldman (SG) [15] potential works well, but the first diamondanvil-cell (DAC) studies showed that it is too stiff, that is the repulsion increases too rapidly with pressure. Hemley et al. $[10,16]$ modified the SG potential [15] with a shortrange correcting term. This Hemley-Silvera-Goldman (HSG) effective potential was shown to fit EOS up to $40 \mathrm{GPa}$, but at higher pressures it is still too stiff [12].

As known, this problem arises because of neglecting of the three body and higher-order terms in the intermolecular potential [14,18-25]. Our many-body potential includes not only pair forces, but triple forces as well. It is a sum of the pair SG potential [15] (discarding the $R^{-9}$ term) and three-body terms which include the long-range AxilrodTeller dispersive interaction and a short-range three-body exchange interaction in the Slater-Kirkwood form [19,21]. It is similar to the potential for solid helium $[19,21]$. An explicit form and parameters of the potential for solid hydrogen are given in Ref. 24.

The calculated pressure-volume semi-empirical dependencies for solid hydrogen and deuterium are given in Ref. 25 in comparison with density-functional theory (DFT)-generalized gradient approximation (GGA) calculations [26] and the main experimental results from $[3,5,7-10,12,13,16]$. It was shown that the semi-empirical (SE) calculations with the proposed many-body potential are in an excellent agreement with experiment in the pres- 
sure range 1-140 GPa (phases I and II). At higher pressure the theoretical SE curve lies slightly below experiment. Above $140 \mathrm{GPa}$ the accuracy of the SE approach decreases with rising pressure, and at the highest reached pressures of $180 \mathrm{GPa}$ (phase III) the difference amounts up to about $10 \%$. At still higher pressure the SE approach progressively underestimate the pressure. The reason for this is supposedly the neglect of the higher order $(n>3)$ terms in the $n$-body expansion of the intermolecular potential. The effect of the large- $n$ terms increases with pressure. Conversely, the accuracy of the EOS from DFT-GGA [26] improves with the increase of pressure: in the pressure range 70-180 GPa the EOS from GGA practically coincides with the experimental one and for $p>140 \mathrm{GPa}$ the agreement is better than for our empirical potentials. At the same time, at lower pressures the $a b$ initio results progressively underestimate the pressure. Thus, the semiempirical and DFT approaches complement each other.

Another experimental technique which provides direct information on intermolecular interactions and vibrational dynamics is Raman scattering. These studies go hand in hand with the EOS studies following the progress in the DAC technique and at present crossed the level of $250 \mathrm{GPa}$. The $E_{2 g}$ Raman frequency $v(P)$ calculated using various empirical potentials is highly sensitive to details of the potential used, making it a hard test for any empirical potential (or for any other theoretical method, e.g., DFT). It is essential that these properties are sensitive to different characteristics of the intermolecular potential: while EOS is sensitive to the potential well depth, the $E_{2 g}$ Raman frequency probes the second derivative of the potential at the minimum.

The hcp structure of solid hydrogen has a Raman-active optical mode ( $E_{2 g}$ symmetry) in the phonon spectrum which corresponds to the out-of-phase shear motions of the two sublattices in the $a b$ plane. The frequency range of this Raman mode is extremely large, from $36 \mathrm{~cm}^{-1}$ at zero pressure [27-36] to $1100 \mathrm{~cm}^{-1}$ at $250 \mathrm{GPa}$. These measurements show that hcp-based structures are stable in this pressure range.

The comparison between theoretical and experimental pressure dependencies $v(P)$ of the $E_{2 g}$ optical phonon Raman-active mode [25] have shown that the phonon Raman modes calculated using various empirical potentials are highly sensitive to details of the potential used, making it a hard test for any empirical potential (or for any other theoretical method, e.g., DFT). The frequencies calculated from the SG potential [15] deviate from experiment even for very low pressures. The same is true [5] for the effective HSG pair potential $[10,16]$. While effective pair potentials work reasonably well for EOS up to $40 \mathrm{GPa}$, they fail for the dynamical properties like Raman spectrum. Similarly to that we had for the EOS, at pressures lower than $\sim 150 \mathrm{GPa}$ the SE curves calculated for the many-body potential [25] agree with experiment better than DFT-LDA (local-density approximation) calculations [25] but at higher pressures the situation is reversed. The limiting pressures at which the SE approach still works are $~ 175$ GPa while the DFT-LDA has a fine agreement with the experiment for $\mathrm{H}_{2}$ from $150 \mathrm{GPa}$ up to the highest considered pressures $~ 230 \mathrm{GPa}[25]$.

One more experimental technique which was used as a source of a critical information on the elastic anisotropy, equation of state, and other thermodynamic properties of this material is measurements of the sound velocity of high-density hydrogen. This information is particularly important for the construction of accurate models for the interior structure of the giant or Jovian planets. Experimental data on sound velocities in solid hydrogen in the pressure range up to $24 \mathrm{GPa}$ were obtained from single-crystal Brillouin scattering experiments [16,37].

The hydrodynamic or bulk sound velocity,

$$
v_{B}=[\partial P / \partial \rho]^{1 / 2}=\left[-\frac{V^{2}}{\mu} \frac{\partial P}{\partial V}\right]^{1 / 2},
$$

where $P$ is pressure, $\rho$ is density, $\mu$ is molar mass, and $V$ is molar volume. On the other hand, the bulk sound velocity, the adiabatic bulk modulus $K_{S}$ and density are connected by the relation

$$
v_{B}=\sqrt{K_{S} / \rho} .
$$

Resulting values of the bulk sound velocity and adiabatic bulk modulus calculated from the obtained SE EOS are shown in Fig. 1. As can be seen, both the bulk sound velocity and adiabatic bulk modulus are in excellent agreement with data from Brillouin scattering measurement [16,37]. As was said above, the empirical SG [27] and

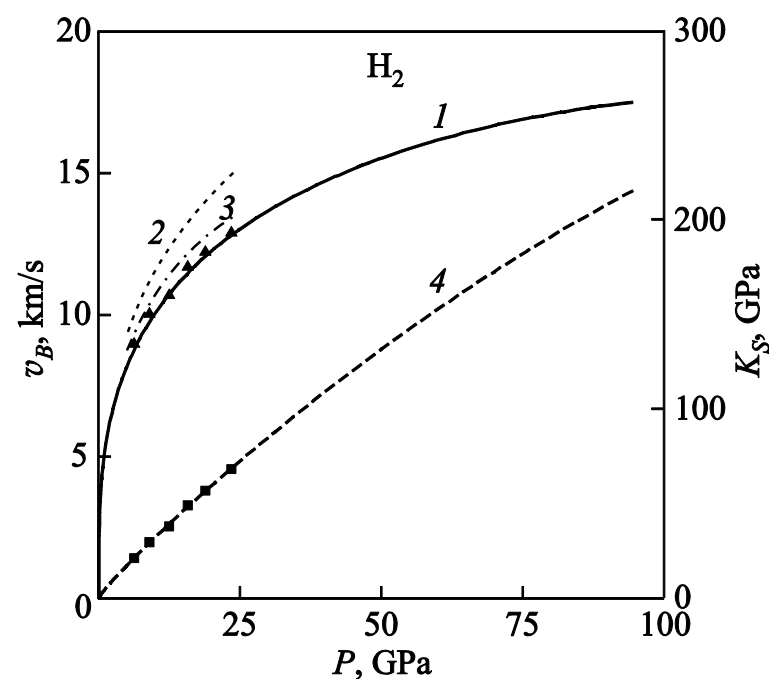

Fig. 1. Bulk sound velocity and adiabatic bulk modulus versus pressure. Sound velocity: calculated from many-body potential (1), from SG potential (2), from HSG potential (3). Solid triangles — experimental data from [16,37] ( $\boldsymbol{\Delta})$. Bulk modulus: calculated from many-body potential (4). Solid squares - experimental data from [16,37] (•). 
HSG [10] potentials potentials for $\mathrm{H}_{2}$ fitted to lower density data, tend to overestimate the repulsive wall of the molecules. The result is an underestimate of the compressibility of dense hydrogen or, equivalently, an overestimate of the sound velocity. It is seen from Fig. 1, the stiffer is the potential, the greater is overestimated the sound velocity.

To make comparison with astrophysical models and data obtained from observations of global oscillations of $\mathrm{Ju}$ piter [38] one need to make predictions of sound velocities over very broad range of pressures where the molecular phase of hydrogen exists, in fact, over $400 \mathrm{GPa}$.

Using first-principle Diffusion Monte-Carlo (DMC) simulations, Alavi et al. [39] calculated the sound velocity of condensed hydrogen to the pressures of $300 \mathrm{GPa}$ (Fig. 2). Their estimated agree well with extrapolated results obtained from Ross-Ree-Young and HSG two-body semi-empirical potentials $[16,17]$. At the same time, the sound velocity obtained from these potentials are markedly higher than the experimental sound velocity [16,17] and the sound velocity calculated from the SE many-body potential. As was said above, the use of this potential is limited by pressures of 140-150 GPa. To have estimates to higher pressure we have also calculated the sound velocity using DFT-LDA approximation (Fig. 2). The LDA sound velocity has been found from the LDA EOS calculated using the full-potential linear muffin-tin orbital (FP-LMTO) code RSPt [40] for the $P c a 2_{1}$ ordered structure - one of the plausible candidates for the orientational structure of phases II and III. Universal EOS parametrization has been used. The LDA approximation apparently overestimates the sound velocity at small pressures and works better for higher pressures.

Combining results from single-crystal Brillouin scattering measurements with synchrotron x-ray diffraction data Zha et al. [37] obtained a complete set of the elastic moduli of solid hydrogen in the pressure range up to $24 \mathrm{GPa}$. Experimental shear elastic modulus and shear sound velocity are given in Fig. 3 as a function of pressure in comparison with theory. Theoretical estimates were obtained from the rela-

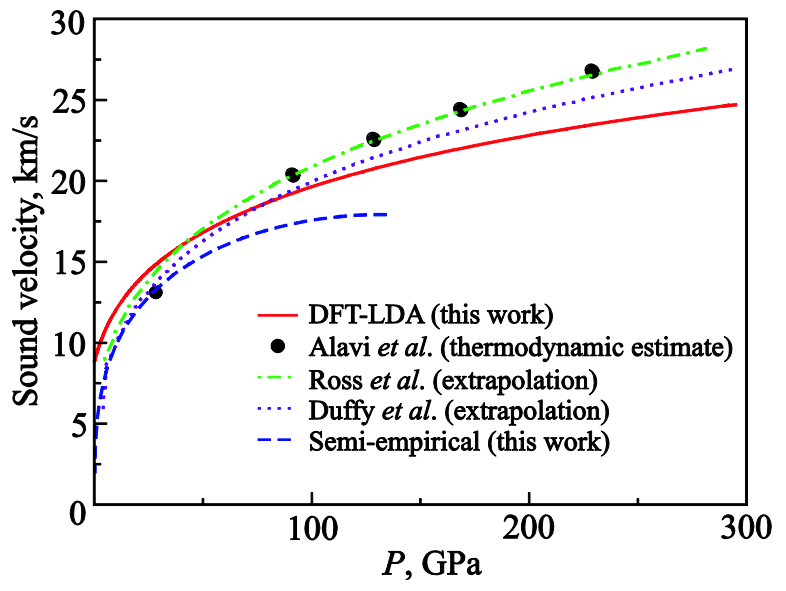

Fig. 2. (Color online) The sound velocities computed using different semi-empirical potentials and first-principle approaches. tion between frequency of the Raman-active $v\left(E_{2 g}\right)$ phonon mode of hcp lattice and elastic constant $C_{44}[41,42]$ :

$$
v\left(E_{2 g}\right)=(2 \pi)^{-1} 4 \sqrt{3} a^{2} C_{44} /(m c)^{1 / 2}
$$

where $a, c$ are the lattice parameters and $m$ is the molecular mass. This relation follows from lattice dynamics studies of the hcp crystal lattice [41] and is exact if the following conditions are met: (a) anharmonic effects are negligible, (b) interactions beyond the next-nearest neighbors are negligible, and (c) four-body and higher manybody interactions are negligible. If some of the conditions (a)-(c) are invalid one might expect that the simple relation between $C_{44}$ and $v\left(E_{2 g}\right)$ breaks down. The applicability of this relation for various hcp metals was examined (see for example Refs. 43 and 44) and it was shown to hold fairly well even though the fulfillment of the conditions (b) and (c) is questionable. For the hcp van der Waals solids (He, $\mathrm{H}_{2}, \mathrm{D}_{2}$ and $\beta-\mathrm{N}_{2}$ ) the same question has been analyzed in Ref. 42. For these substances, especially for solid helium and hydrogens near normal pressure, the most critical is the condition (a). Nonetheless, the departure form the relation was shown to be small. In the absence of direct experimental data such estimates are very useful especially under extreme conditions. In this way we obtain estimates for shear elastic modulus and sound velocity for the lowtemperature molecular phases to extrapolated pressures of $400 \mathrm{GPa}$. We note that the phase III has been shown to be stable to at least $360 \mathrm{GPa}$ at low temperature [45], but hydrogen transforms to other phases (e.g., phase IV) with increasing temperatures at these pressures [46].

In conclusion, we present results of semi-empirical lattice dynamics calculations of the sound velocities in solid hydrogen under pressure based on the many-body intermolecular potential and first-principle density-functional

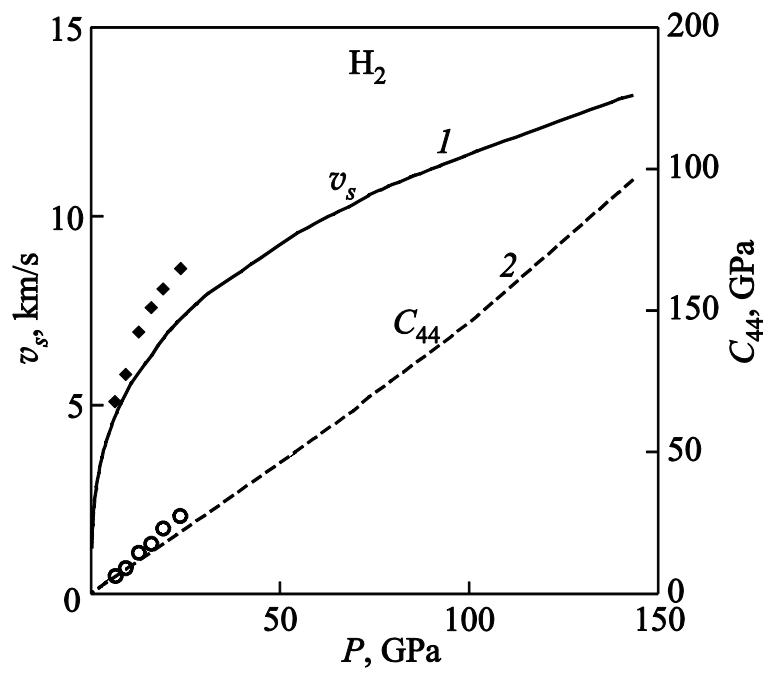

Fig. 3. Shear sound velocity and shear bulk modulus versus pressure. Sound velocity: calculated from many-body potential (1); experimental data from [16,37] $(\bullet)$. Bulk modulus: calculated from many-body potential (2), experimental data from [16,37] (0). 
theory. Both the sound velocities and elastic moduli are in excellent agreement with data from Brillouin scattering measurements while pair SE potentials tend to overestimate the sound velocity. It is shown that the stiffer is the potential the greater is overestimated the sound velocity. As was the case for EOS and Raman-active lattice phonon calculations, the employed many-body potential works well for phases I and II (up to 140 GPa while for higher pressures the use of the DFT is preferable.

This work supported by NSF DMR-1106132. A.F.G. acknowledges support from the NSF, Army Research Office, NAI, and EFRee.

1. M.S. Anderson and C.A. Swenson, Phys. Rev. B 10, 5184 (1974).

2. I.F. Silvera, A. Driessen, and J.A. de Waal, Phys. Lett. A 68 , 207 (1978).

3. A. Driessen, J.A. de Waal, and I.F. Silvera, J. Low Temp. Phys. 34, 255 (1979).

4. I.F. Silvera, Rev. Mod. Phys. 52, 393 (1980).

5. H.-k. Mao and R.J. Hemley, Rev. Mod. Phys. 66, 671 (1994).

6. Physics of Cryocrystals, V.G. Manzhelii and Yu.A. Freiman (eds.) AIP Press, New York (1997).

7. S. Ishmaev, I. Sadikov, A. Chernyshov, B. Vindryaevskii, V. Sukhoparov, A. Telepnev, and G. Kobelev, Sov. Phys. JETP 57, 228 (1983).

8. S. Ishmaev, I. Sadikov, A. Chernyshov, B. Vindryaevskii, V. Sukhoparov, A. Telepnev, G. Kobelev, and R. Sadikov, Sov. Phys. JETP 62, 721 (1985).

9. J. van Straaten and I. Silvera, Phys. Rev. B 37, 1989 (1988).

10. R. Hemley, H.-k. Mao, L. Finger, A. Jephcoat, R. Hazen, and C. Zha, Phys. Rev. B 42, 6458 (1990).

11. S. Besedin, I. Makarenko, S. Stishov, V. Glazkov, I. Goncharenko, A. Irodova, V. Somenkov, and S. Shil'shtein, High-Pressure Res. 4, 447 (1990).

12. P. Loubeyre, R. LeToullec, D. Hausermann, M. Hanfland, R.J. Hemley, H.-k. Mao, and L.W. Finger, Nature 383, 702 (1996).

13. Y. Akahama, M. Nishimura, H. Kawamura, N. Hirao, Y. Ohishi, and K. Takemura, Phys. Rev. B 82, 060101(R) (2010).

14. Yu.A. Freiman, S.M. Tretyak, A. Grechnev, A.F. Goncharov, J.S. Tse, D. Errandonea, H.-k. Mao, and R.J. Hemley, Phys. Rev. B 80, 094112 (2009).

15. I.F. Silvera and V.V. Goldman, J. Chem. Phys. 69, 4209 (1978)

16. T.S. Duffy, W. Vos, C.S. Zha, R.J. Hemley, and H.-k. Mao, Science 263, 1590 (1994).

17. M. Ross, F.H. Ree, and D.A. Young, J. Chem. Phys. 79, 1487 (1983).

18. M. Neumann and M. Zoppi, Phys. Rev. B 62, 41 (2000).

19. P. Loubeyre, Phys. Rev. Lett. 58, 1857 (1987); Phys. Rev. B 37, 5432 (1988).

20. S.M. Tretyak, T.N. Antsygina, and Yu.A. Freiman, Fiz. Nizk. Temp. 32, 1409 (2006) [Low Temp. Phys. 32, 1072 (2006)].
21. Yu.A. Freiman, A.F. Goncharov, S.M. Tretyak, A. Grechnev, J.S. Tse, D. Errandonea, H.-k. Mao, and R.J. Hemley, Phys. Rev. B 78, 014301 (2008).

22. H. Shimizu, M. Kawajiri, T. Kume, S. Sasaki, Yu.A. Freiman, and S.M. Tretyak, Phys. Rev. B 79, 132101 (2009).

23. A. Grechnev, S.M. Tretyak, and Yu.A. Freiman, Fiz. Nizk. Temp. 36, 423 (2010) [Low Temp. Phys. 36, 333 (2010)].

24. Yu.A. Freiman, S.M. Tretyak, Alexander F. Goncharov, H.-k. Mao, and R.J. Hemley, Fiz. Nizk. Temp. 37, 1302 (2011) [Low Temp. Phys. 37, 1038 (2011)].

25. Yu.A. Freiman, A. Grechnev, S.M. Tretyak, Alexander F. Goncharov, and R.J. Hemley, Phys. Rev. B 86, 014111 (2012).

26. L.J. Zhang, Y.L. Niu, T. Cui, Y. Li, Y. M. Ma, Z. He, and G.T. Zou, J. Phys.: Condens. Matter 19, 425237 (2007).

27. I.F. Silvera, W.N. Hardy, and J.P. McTague, Phys. Rev. B 5, 1578 (1972).

28. M. Nielsen, Phys. Rev. B 7, 1626 (1973).

29. P.J. Berkhout and I.F. Silvera, J. Low Temp. Phys. 36, 231 (1979).

30. R.J. Wijngaarden, V.V. Goldman, and I.F. Silvera, Phys. Rev. B 27, 5084 (1983).

31. A. Lagendijk, R.J. Wijngaarden, and I.F. Silvera, Phys. Rev. B 31, 1352 (1985).

32. M. Hanfland, R.J. Hemley, and H.-k. Mao, High-Pressure Science and Technology - 1993, S.C. Schmidt et al. (eds.) AIP, New York (1994), p. 877.

33. R.J. Hemley, H.-k. Mao, and J. Shu, Phys. Rev. Lett. 65 , 2670 (1990).

34. A.F. Goncharov, R.J. Hemley, H.-k. Mao, and J. Shu, Phys. Rev. Lett. 80, 101 (1998).

35. A.F. Goncharov, E. Gregoryanz, R.J. Hemley, and H.-k. Mao, PNAS 98, 14234 (2001).

36. R.J. Hemley, J.H. Eggert, and H.-k. Mao, Phys. Rev. B 48 , 5779 (1993).

37. C.S. Zha, T.S. Duffy, H.-k. Mao, and R.J. Hemley, Phys. Rev. B 48, 9246 (1993).

38. B. Mosser, F.X. Schmider, Ph. Delache, and D. Gautier, A\&A 251, 356 (1991).

39. A. Alavi, M. Parrinello, and D. Frenkel, Science 269, 1252 (1995).

40. J.M. Wills, M. Alouani, P. Andersson, A. Delin, O. Eriksson, and O. Grechnyev, Full-Potential Electronic Structure Method: Energy and Force Calculations with Density Functional and Dynamical Mean Field Theory, Springer, Berlin (2010).

41. E.A. Metzbower, Phys. Status Solidi 25, 403 (1968).

42. H. Olijnyk and A.P. Jephcoat, J. Phys.: Condens. Matter 12, 10423 (2000).

43. H. Olijnyk and A.P. Jephcoat, Solid State Commun. 115, 335 (2000).

44. S. Merkel, A.F. Goncharov, H.-k. Mao, P. Gillet, and R.J. Hemley, Science 288, 1626 (2000).

45. C.S. Zha, Z. Liu, and R.J. Hemley, Phys. Rev. Lett. 108, 146402 (2012).

46. C.S. Zha, Z. Liu, M. Ahart, R. Boehler, and R.J. Hemley, to be published. 